\title{
Efficacy of percutaneous coronary intervention in diabetic patients - local results with global impact
}

\author{
Hrvoje Jurin*, Aleksander Ernst, Joško Bulum, Maja Strozzi \\ University of Zagreb School of Medicine, University Hospital Center Zagreb, Zagreb, Croatia
}

Introduction: Diabetes mellitus is combined with high risk for the development of coronary artery disease (CAD). Revascularization in diabetic patients is challenged by a more diffuse atherosclerotic coronary disease, a higher propensity to develop re-stenosis and unremitting atherosclerotic progression causing new stenosis.

The goal: To assess efficacy, safety and long-term survival of diabetic patients with mutivessel CAD treated with percutaneous coronary intervention (PCI) and drug-eluting stent implantation (DES).

Patients and Methods: We included 28 patients with stable/unstable angina or non-ST segment elevation myocardial infarction (age 66 615 ) and with basal characteristics and inherent risk comorbidities as stated in Table 1. After the coronarography was done and multivessel CAD was confirmed (significant stenosis in more than 2 epicardial vessels and/or stenosis of the left main coronary artery) patients were offered, regarding optimal evidence-based medical possibilities, surgical revascularization or $\mathrm{PCl}$. The latter ones entered the $\mathrm{PCl}$ arm of the study and were treated with $\mathrm{PCl}$ using DES. After the patients underwent coronarography,

Received: $20^{\text {th }}$ Apr 2014

*Address for correspondence: Klinički bolnički centar Zagreb, Kišpatićeva 12, HR10000 Zagreb, Croatia.

Phone: +385-98-525-002

E-mail: hrvoje.jurin@gmail.com
SYNTAX score was calculated which classifies patients into low, medium and high SYNTAX score group (SYNTAX score $<22,23-32$ and $>33$ ). Following $\mathrm{PCl}$, all the patients were treated by using optimal medical therapy (Table 2). The patients were subjected to secondary coronarography after one year of follow-up or earlier based on clinical indication.

Results: After the median follow-up of 3.4 years, we found that the highest incidence of major adverse cardiac and cerebrovascular event (MACCE) was observed in high SYNTAX score group (100\%) as opposed to absence of MACCE in the low SYNTAX score group (Table 3). The need for repeated revascularization was $14.3 \%$ and the overall registered primary outcome prevalence (composite of death, myocardial infarction and stroke) was $21.4 \%$. None of the patients had stroke and the incidence of myocardial infarction was $14.2 \%$ (all observed in high SYNTAX score group).

Conclusion: The results of our study are comparable to the results of large randomized clinical trials conducted worldwide which studied the efficacy of $\mathrm{PCl}$ in multivessel disease as well as compared $\mathrm{PCl}$ strategy with surgical revascularization. Therefore, we find that these results indicate the possibilities of implementing and performing "state-of-theart" cardiovascular procedures even in somewhat small clinical centers as ours whilst obtaining results comparable to the ones achieved in large global centers.

KEYWORDS: diabetes, multivessel coronary artery disease, drug-eluting stent.

CITATION: Cardiol Croat. 2014;9(5-6):179-180.

Table 1. Baseline characteristics, comorbidities and calculated SYNTAX score in the group of patients treated with percutaneous coronary intervention and drug-eluting stent implantation.

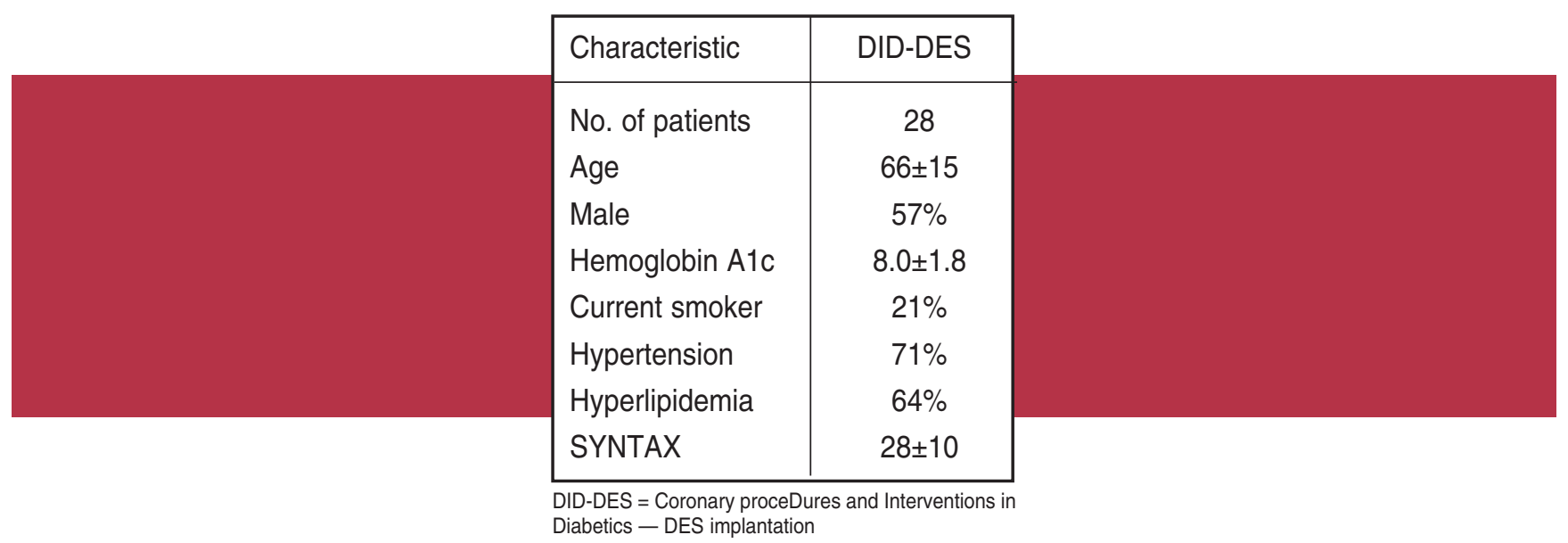


Table 2. Percentage of patients receiving optimal medications after percutaneous coronary intervention.

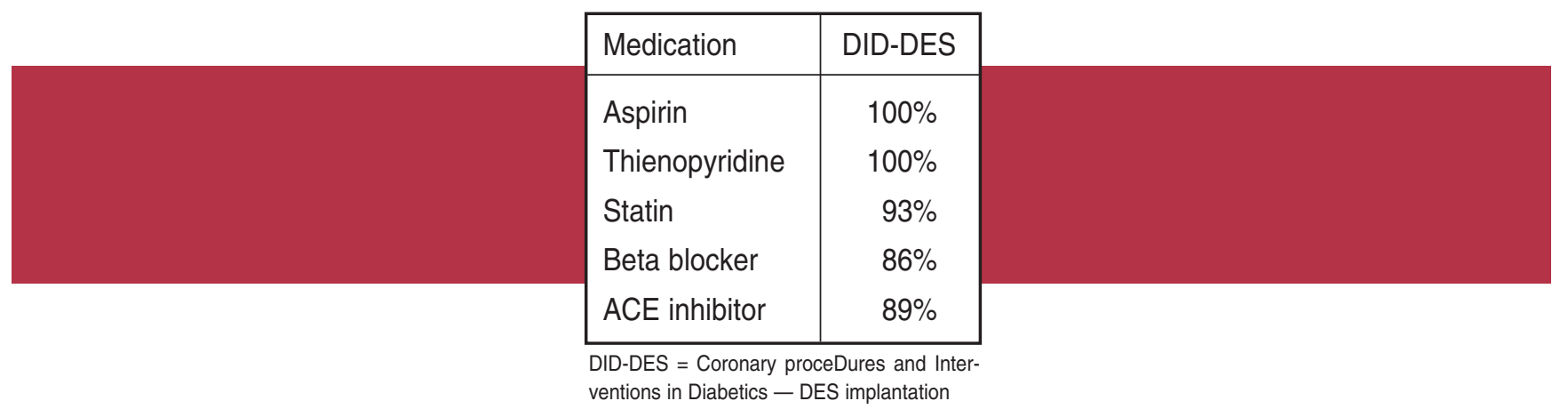

Table 3. Final results after median follow-up of 3.4 years.

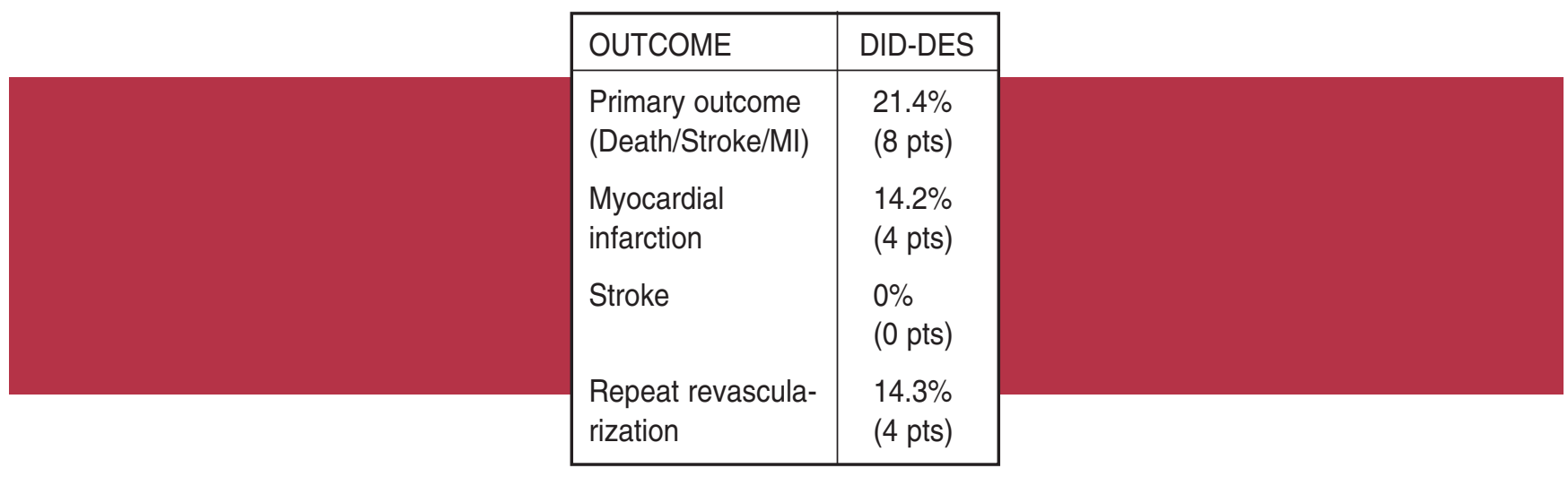

DID-DES = Coronary proceDures and Interventions in Diabetics - DES implantation

\section{Literature}

1. Farkouh ME, Domanski M, Sleeper LA, et al; FREEDOM Trial Investigators. Strategies for multivessel revascularization in patients with diabetes. N Engl J Med. $2012 ; 367: 2375-84$. 2. Lim JY, Deo SV, Kim WS, Altarabsheh SE, Erwin PJ, Park SJ. Drug-eluting stents versus coronary artery bypass grafting in diabetic patients with multi-vessel disease: a metaanalysis. Heart Lung Circ. 2014 Feb 26. pii: S1443-9506(14)00075-4. doi: 10.1016/j.hlc.2014.02.005. [Epub ahead of print].

3. De Luca G, Schaffer A, Verdoia M, Suryapranata H. Meta-analysis of 14 trials comparing bypass grafting vs drug-eluting stents in diabetic patients with multivessel coronary artery disease. Nutr Metab Cardiovasc Dis. 2014;24(4):344-54.

4. Jurin $\mathrm{H}$, Ernst A, Bulum J, Strozzi M. Long-term survival of diabetic patients with multivesel coronary artery disease treated with percutaneous coronary intervention and drugeluting stents implantation - our experiences. Cardiol Croat. 2013;8(3-4):114. 